REPLY

\section{Type 1 diabetes mellitus is a heterogeneous disease}

\section{Mikael Knip}

I would like to thank Teresa Rodriguez-Calvo, Mark Atkinson and Matthias von Herrath for their correspondence on my News \& Views article (Loss of $\beta$-cell mass - an acute event before T1DM presentation? Nat. Rev. Endocrinol. 13, 253-254 (2017)) ${ }^{1}$, which raised some important questions ( $\beta$-Cell mass versus function in type 1 diabetes mellitus: truth or dare? Nat. Rev. Endocrinol. http://dx.doi. org/10.1038/nrendo.2017.83 (2017) $)^{2}$. In their correspondence ${ }^{2}$, Rodriguez-Calvo and colleagues emphasized that type 1 diabetes mellitus (T1DM) is a heterogeneous disease I definitely agree with that statement. One sign of the heterogeneity in T1DM is the conspicuous variation between individuals with respect to the duration of asymptomatic preclinical disease. The fastest course from autoantibody seroconversion to the appearance of clinical symptoms of T1DM that we have seen was 2 months in an infant; however, the longest interval we have observed was in a female, who seroconverted at the age of 11 years and presented with overt T1DM at the of 32 years ${ }^{3}$.

Rodriguez-Calvo et al. ${ }^{2}$ argue again that their finding of intact $\beta$-cell mass in 13 autoantibody-positive organ donors from the Pancreatic Organ Donors with Diabetes (nPOD) study provides direct evidence that the loss of $\beta$-cell mass is an acute event before T1DM presentation. I still disagree that this statement is true in most patients. For obvious reasons, there is no available data regarding $\beta$-cell mass in the same individual close to and after the diagnosis of T1DM. There are a series of facts that speak against the assertion that the autoantibody-positive nPOD donors studied would have been close to the diagnosis of T1DM. In a study in children derived from the general population and with a mean age of 9 years, only $26 \%$ ( 9 of 34 ) of children who tested positive for glutamic acid decarboxylase (GAD) autoantibodies progressed to clinical T1DM during a 26-year follow-up period $^{3}$. The mean duration of the preclinical period was 8.5 years in those cases. All seven $(21 \%)$ children who tested positive for both GAD autoantibodies and tyrosine phosphatase IA2 (IA2; also known as ICA512) autoantibodies initially progressed to overt disease, but only after a mean observation period of 7.7 years. It is well established that the rate of progression from autoantibody positivity to clinical disease is faster in children than in adults. Indeed, the mean age of the autoantibody-positive nPOD donors was 36 years in their Diabetes paper ${ }^{4}$. Collectively, these data imply that only a minority of the autoantibody-positive nPOD donors would have progressed to T1DM, and, if so, T1DM would have presented after several years.

It is conceivable that the loss of $\beta$-cell mass might be an acute event in some individuals who present with clinical T1DM; for example, in those with disease manifestation between the ages of 6 and 12 months. However, direct evidence for an acute loss of $\beta$-cell mass before the diagnosis of T1DM is still missing - an old dogma can hardly be dismissed by establishing a new one.

Mikael Knip is at the Children's Hospital, Department of Paediatrics, University of Helsinki and Helsinki University Hospital, PO Box 22, Fl-00014 Helsinki, Finland. mikael.knip@helsinki.fi

1. Knip, M. Loss of $\beta$-cell mass: an acute event before T1DM presentation? Nat. Rev. Endocrinol. 13 253-254 (2017).

2. Rodriguez-Calvo, T. et al. $\beta$-Cell mass versus function in type 1 diabetes mellitus: truth or dare? Nat. Rev. Endocrinol. http://dx.doi.org/10.1038/ nrendo.2017.83 (2017).

3. Knip, M. et al. Prediction of type 1 diabetes in the general population. Diabetes Care 33, 1206-1212 (2010).

4. Rodriguez-Calvo, T. et al. Increase in pancreatic proinsulin and preservation of $\beta$-cell mass in autoantibody positive donors prior to type 1 diabetes onset. Diabetes 66, 1334-1345 (2017).

Competing interests statement

M.K. declares that he has received a lecture fee from Merck Sharpe $\&$ Dohme (MSD), and that he is a board member and minor shareholder $(<5 \%)$ of Vactech Oyj. 